\title{
Linearly degenerate hierarchies of quasiclassical SDYM type
}

\author{
L.V. Bogdanov ${ }^{* 1}$ and M.V. Pavlov ${ }^{2,3,4}$ \\ ${ }^{1}$ L.D. Landau ITP, Moscow \\ ${ }^{2}$ Lebedev Physical Institute, Moscow \\ ${ }^{3}$ National Research Nuclear University MEPHI, Moscow \\ ${ }^{4}$ Department of Mechanics and Mathematics, Novosibirsk State \\ University, Novosibirsk, Russia
}

\begin{abstract}
We demonstrate that SDYM equations for the Lie algebra of onedimensional vector fields represent a natural reduction in the framework of general linearly degenerate dispersionless hierarchy. We define the reduction in terms of wave functions, introduce generating relation, Lax-Sato equations and the dressing scheme for the reduced hierarchy. Multidimensional case is also discussed.
\end{abstract}

\section{Introduction}

This work is connected with the class of equations containing quasiclassical self-dual Yang-Mills equations. Let us consider a Lax pair

$$
\begin{aligned}
& L=\partial_{t_{1}}-\lambda \partial_{y_{1}}+A_{1}, \\
& M=\partial_{t_{2}}-\lambda \partial_{y_{2}}+A_{2},
\end{aligned}
$$

where $A_{1}, A_{2}$ belong to some Lie algebra, $\lambda$ is a complex variable (spectral parameter). Commutativity condition for operators (1) implies the existence of potential $F, A_{1}=\partial_{y_{1}} F, A_{2}=\partial_{y_{2}} F$, satisfying the equation

$$
\partial_{t_{1}} \partial_{y_{2}} F-\partial_{t_{2}} \partial_{y_{1}} F-\left[\partial_{y_{1}} F, \partial_{y_{2}} F\right]=0
$$

*leonid@itp.ac.ru 
For the case of matrix Lie algebra this is a well-known (complexified) selfdual Yang-Mills equation (see, e.g., 1], 2]). For the case of Lie algebra of vector fields, $F=\sum_{i=1}^{N} v^{i} \partial_{x_{i}}$, equation (2) represents $(N+4)$-dimensional 'quasiclassical' self-dual Yang-Mills equations for the coefficients of vector field $F$ (see [3], 4])

$$
\partial_{t_{1}} \partial_{y_{2}} v^{i}-\partial_{t_{2}} \partial_{y_{1}} v^{i}-\sum_{j=1}^{N}\left(\partial_{y_{1}} v^{j}\right) \partial_{x_{j}} \partial_{y_{2}} v^{i}+\sum_{j=1}^{N}\left(\partial_{y_{2}} v^{j}\right) \partial_{x_{j}} \partial_{y_{1}} v^{i}=0
$$

where $1 \leqslant i \leqslant N$, which for two-dimensional Hamiltonian vector fields reduces to six-dimensional heavenly equation [3].

In this work we will consider mainly one-dimensional vector fields, $F=$ $v \partial_{x}$, then linear equations corresponding to Lax pair (1) are

$$
\begin{aligned}
& \partial_{t_{1}} \Psi=\left(\lambda \partial_{y_{1}}-\left(\partial_{y_{1}} v\right) \partial_{x}\right) \Psi, \\
& \partial_{t_{2}} \Psi=\left(\lambda \partial_{y_{2}}-\left(\partial_{y_{2}} v\right) \partial_{x}\right) \Psi,
\end{aligned}
$$

and compatibility conditions (3) take the form of one five-dimensional equation

$$
\partial_{t_{1}} \partial_{y_{2}} v-\partial_{t_{2}} \partial_{y_{1}} v-\left(\partial_{y_{1}} v\right)\left(\partial_{x} \partial_{y_{2}} v\right)+\left(\partial_{y_{2}} v\right)\left(\partial_{x} \partial_{y_{1}} v\right)=0
$$

Performing dimensional reduction $\partial_{y_{1}}=\partial_{x}, \partial_{t_{1}}=\partial_{y_{2}}\left(=\partial_{y}\right)$ (respectively, considering $\left.\Psi=\Psi\left(\lambda, x+y_{1}, t_{1}+y_{2}, t_{2}=t\right)\right)$, we come to linear equations

$$
\begin{aligned}
& \partial_{y} \Psi=\left(\lambda \partial_{x}-v_{x} \partial_{x}\right) \Psi, \\
& \partial_{t} \Psi=\left(\lambda \partial_{y}-v_{y} \partial_{x}\right) \Psi
\end{aligned}
$$

corresponding to $(2+1)$-dimensional equation [5]

$$
v_{t x}=v_{y y}+v_{x} v_{x y}-v_{y} v_{x x},
$$

which is a simple representative of a class of linearly degenerate (or weakly nonlinear) dispersionless integrable equations (see [6], [7, [8])), connected with the so-called universal hierarchy of Shabat and Martinez Alonso [9]. A more general geometric setting for the reduction of $\operatorname{Diff}\left(S^{1}\right) \mathrm{SDYM}$ equations to equation (7) was presented in [10].

Our main purpose is a description of the hierarchy connected with equations (44) and its generalizations in the framework of general multidimensional dispersionless hierarchy, using the technique developed in [11, [12], [13. We will demonstrate that hierarchies of this type represent a rather 
natural reduction of generic linearly degenerate dispersionless case. We will also discuss an immersion of 'universal' hierarchy of Shabat and Martinez Alonso [9] to multidimensional hierarchies connected with equations of the type (4), (5).

A natural language to consider multiple commuting flows or the whole hierarchy for equations connected with commuting vector fields is a language of distributions. A distribution $\Delta$ is an $K$-dimensional subbundle of the tangent bundle of smooth $N$-dimensional manifold, possessing a basis of $K$ smooth vector fields $V_{1}, \ldots V_{K}$. Taking local coordinates $x_{1}, \ldots, x_{N}$, we can write the basis as $V_{i}=\sum_{k=1}^{N} v_{i}^{k} \partial_{k}, \partial_{k}=\frac{\partial}{\partial x_{k}}$. A distribution $\Delta$ is called involutive if $[\Delta, \Delta] \subset \Delta$ (in the sense of commutation of vector fields). According to Frobenius theorem for vector fields, for the case of involutive distributions a system of partial differential equations

$$
V_{i} f:=\sum_{k=1}^{N} v_{i}^{k} \partial_{k} f=0, \quad 1 \leqslant i \leqslant K
$$

locally admits $M=N-K$ (we will call $M$ a codimension of the distribution) independent solutions $f_{1}, \ldots, f_{M}$. After some renotation of variables, the basic vector fields can always be chosen in a form

$$
V_{i}=\frac{\partial}{\partial t_{i}}-\sum_{k=1}^{M} v_{i}^{k} \partial_{k}, \quad 1 \leqslant i \leqslant K
$$

where local variables are now $t_{1}, \ldots, t_{K}, x_{1}, \ldots, x_{M}$. For the involutive (integrable) case the basic vectors $V_{i}$ commute, each subset of basic vectors gives a subdistribution with the same codimension (in remaining variables), and the Jacobian of functions $f_{1}, \ldots, f_{M}$ over variables $x_{1}, \ldots, x_{M}$ is not equal to zero. The distribution is tangent to the level surfaces of the functions $f_{1}, \ldots, f_{M}$ and is uniquely defined by these functions.

The basis of the form (9) is a good starting point to consider the system of commuting flows, where the variables $t_{1}, \ldots, t_{K}$ are 'times' of the flows. One more ingredient we need to consider dispersionless integrable equations is holomorphicity. We suggest that coefficients of the basis $v_{i}^{k}$ depend holomorpically on the spectral variable $\lambda$. In the present work, we consider mostly the simplest case of polynomial coefficients. Then commutativity (or, more generally, involutivity) of vector fields provides equations for the coefficients of polynomials. Solutions of equations (8) $f_{1}, \ldots, f_{M}$ depend in this case on the spectral parameter, and we call them wave fuctions (using the terminology common in the theory of integrable equations). A general wave function is given by the expression $\Psi(\lambda, \mathbf{x})=F\left(\lambda, f_{1}(\lambda, \mathbf{x}), \ldots, f_{M}(\lambda, \mathbf{x})\right)$, and 
the transformation between two sets of basic wave functions is defined by $M$ functions of $M+1$ variables. This transformation defines functional freedom in the Riemann-Hilbert problem (see below) and indicates that (maximal) dimensionality of integrable equations corresponding to the distribution of codimension $M$ is $M+2$.

Let us consider linear equations (6) from this viewpoint. These equations correspond to basic vectors of two-dimensional distribution in threedimensional space of variables $x, y, t$. Involutivity is provided by equation (17), and, according to Frobenius theorem, equations (6) admit one independent solution (wave function) $\Psi(\lambda, x, y, t)$, and a general solution is given locally by the function $F(\Psi, \lambda)$. The function $\Psi$ can be found in the form of series in $\lambda$,

$$
\Psi=\Psi_{0}+\widetilde{\Psi}, \quad \Psi_{0}=\lambda^{2} t+\lambda y+x, \quad \widetilde{\Psi}=\sum_{n=1}^{\infty} \Psi_{n}(x, y, t) \lambda^{-n},
$$

where the coefficients $\Psi_{n}(x, y, t)$ are defined recursively through linear equations (6). Linear equations (6) can be also rewritten in the form corresponding to standard basis structure (9),

$$
\begin{aligned}
\partial_{y} \Psi & =\left(\lambda \partial_{x}-v_{x} \partial_{x}\right) \Psi, \\
\partial_{t} \Psi & =\left(\lambda^{2} \partial_{x}-\lambda v_{x} \partial_{x}-v_{y} \partial_{x}\right) \Psi,
\end{aligned}
$$

which can be extended to the infinite hierachy of the form

$$
\partial_{t_{n}} \Psi=\left(\lambda^{n}-P_{n-1}\right) \partial_{x} \Psi, \quad 1 \leqslant n<\infty,
$$

where $P_{n-1}$ are polynomials in $\lambda$ of the order $n-1$ with the coefficients depending on $x$ and times. Solution (10) retains its structure, with $\Psi_{0}$ generalized to the infinite set of times,

$$
\Psi=\Psi_{0}+\widetilde{\Psi}, \quad \Psi_{0}=\sum_{k=0}^{\infty} \lambda^{k} t_{k}, \quad \widetilde{\Psi}=\sum_{n=1}^{\infty} \Psi_{n}(\mathbf{t}) \lambda^{-n},
$$

and $x=t_{0}, y=t_{1}, t=t_{2}$. Codimension of correspondinq distribution is 1 for any restriction to the finite set of times, basic vectors for the case of involutive distribution are commuting and commutation condition for any pair of them gives a closed (2+1)-dimensional system for the coefficients of corresponding polynomials. The hierarchy can be also written in recursive form, which continues the basic set of the form (6),

$$
\partial_{t_{n}} \Psi=\left(\lambda \partial_{t_{n-1}}-v_{t_{n-1}} \partial_{x}\right) \Psi, \quad 1 \leqslant n<\infty, x=t_{0} .
$$


This hierarchy is a simplest example of linearly-degenerate dispersionless hierarchy, it corresponds to the so-called universal hierarchy of Shabat and Martinez Alonso [9] for positive times. The picture of the hierarchy can be developed starting from the wave function in the form of series (12) and formulating the dynamics in terms of the series, or, equivalently, in terms of hydrodynamic type chains $[5]$.

Let us proceed now to the initial SDYM equation for the Lie algebra of one-dimensional vector fields (five-dimensional quasiclassical SDYM equation) (4), (5). Codimension of the distribution is three, and equations (44) should have three independent solutions. One of these solutions is of the form (10),

$$
\Psi=x+\sum_{n=1}^{\infty} \Psi_{n}(\mathbf{t}) \lambda^{-n},
$$

and two others are trivial,

$$
\Psi^{1}=\lambda t_{1}+y_{1}, \quad \Psi^{2}=\lambda t_{2}+y_{2} .
$$

In [12], 13] we developed a picture of general multidimensional dispersionless hierarchy corresponding to $N$ wave functions of the form (12) with $N$ infinite sets of times. From this viewpoint, equation (5) is connected with a special reduction of general linearly degenerate 5-dimensional dispersionless hierarchy, characterised by the condition $\Psi^{1}=\Psi_{0}^{1}, \Psi^{2}=\Psi_{0}^{2}$. Thus two of the wave functions are analytic (polynomial for finite set of variables). In terms of initial Lax pair (11) the general case corresponds to the presence of generic terms with derivatives $\partial_{y_{1}}, \partial_{y_{2}}$ in both vector fields $A_{1}$, $A_{2}$ (and then in $F$ ), that corresponds to canonical structure of general basis for the distribution (9). In the reduced case some of the coefficients of vector fields are equal to zero, and reduction is characterised by the presence of analytic (polynomial) wave functions. The functional freedom in the Riemann-Hilbert problem for the reduced case is one function of four variables (see below), that corresponds to the fact that the reduction is not dimensional, and the reduced systems preserves the dimensionality five.

There exists an interesting direct immersion of $(2+1)$-dimensional hierarchy (11), (13) to the five-dimensional system (5), (41). Indeed, let us consider a pair of linear equations (13) corresponding to two distinct pairs of times $t_{n}, t_{n-1}$ and $t_{k}, t_{k-1}$,

$$
\begin{aligned}
& \partial_{t_{n}} \Psi=\left(\lambda \partial_{t_{n-1}}-v_{t_{n-1}} \partial_{x}\right) \Psi, \\
& \partial_{t_{k}} \Psi=\left(\lambda \partial_{t_{k-1}}-v_{t_{k-1}} \partial_{x}\right) \Psi
\end{aligned}
$$


This system exactly corresponds to linear system (4). Thus, having a dependence of solution of the (2+1)-dimensional hierarchy (11) on two arbitrary distinct pairs of higher times, we get a special solution to five-dimensional equation (5). Thus equation (5) plays a role of 'intertwiner' for higher flows of the hierarchy associated with the distribution (11), (13). A similar phenomenon is known for the case of SDYM hierarchy [1].

\subsection{Towards general multidimensional case}

Let us consider first a simple characteristic example which demonstrates some distinctive features of quasiclassic SDYM case, connected with the fact that corresponding distributions are rather special, starting from threedimensional distribution with the basic equations

$$
\begin{aligned}
\partial_{t_{1}} \Psi & =\left(\lambda \partial_{y_{1}}-v_{y_{1}} \partial_{x}\right) \Psi, \\
\partial_{t_{2}} \Psi & =\left(\lambda \partial_{y_{2}}-v_{y_{2}} \partial_{x}\right) \Psi, \\
\partial_{t_{3}} \Psi & =\left(\lambda \partial_{y_{3}}-v_{y_{3}} \partial_{x}\right) \Psi .
\end{aligned}
$$

Codimension of this distribution is 4 , and we expect integrable systems of (maximal) dimension 6. However, taking compatibility condition for any pair of equations (15), we get equations of dimension 5. This phenomenon is connected with presence of polynomial solutions $\Psi^{1}=\lambda t_{1}+y_{1}, \Psi^{2}=$ $\lambda t_{2}+y_{2}, \Psi^{3}=\lambda t_{3}+y_{3}$ (the fourth function of the basic set is generic, of the form (14)). Lax pair for some integrable equation is obtained from the distribution (containing several commuting flows) by restriction to some subset of variables (or, more generally, by restriction to some submanifold) to obtain two-dimensional distribution, which is especially simple for the basis of the form (9). For the case of generic wave functions, the number of wave functions and respectively the codimension of the distribution is preserved, and we get $R+2$-dimensional equations. However, if there exist special wave functions independent on some of the variables (like in the present example), they may drop out in the process of restriction of the set of variables, then the codimension of the distribution and respectively the dimensionality of the system will be less then maximal.

One of the ways to get 5 -dimensional system in this case is a restriction of the set of variables. Let us consider a restriction to submanifold $x=$ const. The distribution in this case is two-dimensional and consists of the vectors, not containing $\partial_{x}$, the basis can be obtained from (15), taking appropriate linear combinations. In terms of the basic equations, we can express $\partial_{x} \Psi$ 
from the first equation in (15),

$$
\partial_{x} \Psi=-\frac{1}{v_{y_{1}}}\left(\partial_{t_{1}}-\lambda \partial_{y_{1}}\right) \Psi .
$$

Substituting this expression into third and and second equations of the system (15), we obtain

$$
\begin{aligned}
\left(\partial_{t_{2}}-\lambda \partial_{y_{2}}\right) \Psi & =\frac{v_{y_{2}}}{v_{y_{1}}}\left(\partial_{t_{1}}-\lambda \partial_{y_{1}}\right) \Psi \\
\left(\partial_{t_{3}}-\lambda \partial_{y_{3}}\right) \Psi & =\frac{v_{y_{3}}}{v_{y_{1}}}\left(\partial_{t_{1}}-\lambda \partial_{y_{1}}\right) \Psi
\end{aligned}
$$

the compatibility condition for this system (the commutativity of the basic vectors of the distribution) gives a quasilinear equations of second order,

$$
v_{y_{3}}\left(v_{y_{2} t_{1}}-v_{y_{1} t_{2}}\right)+v_{y_{1}}\left(v_{y_{3} t_{2}}-v_{y_{2} t_{3}}\right)+v_{y_{2}}\left(v_{y_{1} t_{3}}-v_{y_{3} t_{1}}\right)=0 .
$$

Remark. This six-dimensional quasilinear equation of the second order is connected with the two-component first order quasilinear system [14]

$$
\begin{aligned}
& f g_{y_{1}}-g f_{y_{1}}+f_{y_{3}}-g_{y_{2}}=0, \\
& g f_{t_{1}}-f g_{t_{1}}+g_{t_{2}}-f_{t_{3}}=0,
\end{aligned}
$$

associated with a Lax pair

$$
\begin{aligned}
\left(\partial_{t_{2}}-\lambda \partial_{y_{2}}\right) \Psi & =f\left(\partial_{t_{1}}-\lambda \partial_{y_{1}}\right) \Psi \\
\left(\partial_{t_{3}}-\lambda \partial_{y_{3}}\right) \Psi & =g\left(\partial_{t_{1}}-\lambda \partial_{y_{1}}\right) \Psi
\end{aligned}
$$

linear system (16) and equation (17) are obtained by the substitution [15]

$$
f=\frac{v_{y_{2}}}{v_{y_{1}}}, \quad g=\frac{v_{y_{3}}}{v_{y_{1}}} .
$$

Another way is to make a restriction to a slightly more involved submanifold, the advantage of this way is that it can be easily generalized to an arbitrary number of the basic equations of the form (15). Let us consider a restriction of the distribution (15) to submanifold $y_{2}=t_{3}=\tau$. Then we get 2-dimensional distribution in the space of 6 variables $t_{1}, y_{1}, t_{2}, \tau, y_{3}, x$ with a basis

$$
\begin{aligned}
\partial_{t_{1}} \Psi & =\left(\lambda \partial_{y_{1}}-\left(\partial_{y_{1}} v\right) \partial_{x}\right) \Psi \\
\partial_{t_{2}} \Psi & =\left(-\lambda^{2} \partial_{y_{3}}+\lambda \partial_{\tau}+\left(\lambda\left(\partial_{y_{3}} v\right)+u\right) \partial_{x}\right) \Psi
\end{aligned}
$$


and a compatibility condition gives a six-dimensional system of equations for two functions $u, v$,

$$
\begin{array}{r}
u_{y_{1}}-v_{y_{3} t_{1}}-v_{y_{1} \tau}+v_{y_{1}} v_{y_{3} x}-v_{y_{1} x} v_{y_{3}}=0, \\
v_{y_{1} t_{2}}-u_{t_{1}}+v_{x y_{1}} u-v_{y_{1}} u_{x}=0 .
\end{array}
$$

The considered example gives us some insight into the structure of general multidimensional quasiclassical SDYM hierarchy for the Lie algebra of one-dimensional vector fields. We will now give a sketch of general picture, which will be developed in more detail below.

For the case of $(M+2)$-dimensional hierarchy, $M \geqslant 3$, corresponding to distribution of codimension $M$ (and, respectively, equations of maximal dimensionality $M+2$ ) we have $M-1$ infinite series of variables $t_{n}^{k}, 1 \leqslant k \leqslant$ $M-1,1 \leqslant n<\infty$ and a variable $x$ (one-dimensional vector field variable). The hierarchy is defined by linear equations

$$
\partial_{t_{n+1}^{k}} \Psi=\left(\lambda \partial_{t_{n}^{k}}-\left(\partial_{t_{n}^{k}} v\right) \partial_{x}\right) \Psi, \quad 1 \leqslant k \leqslant M-1, \quad 1 \leqslant n<\infty,
$$

corresponding to the basic vectors of the distribution in recursive form. Equations (19) have $M-1$ analytic (polynomial for finite number of times) solutions

$$
\Psi^{k}=\sum_{n=0}^{\infty} t_{n+1}^{k} \lambda^{n}
$$

and one solution of the form (14),

$$
\Psi=x+\sum_{n=1}^{\infty} \Psi_{n}(\mathbf{t}, x) \lambda^{-n} .
$$

To obtain equations of maximal dimensionality $M+2$ in the framework of this hierarchy, it is possible to apply the scheme of restriction to the submanifold used to derive system (18), which is easily generalized to the multidimensional case. The structure of the Lax pair in this case is

$$
\begin{aligned}
\partial_{t_{1}} \Psi & =\left(\lambda \partial_{y_{1}}-\left(\partial_{y_{1}} v\right) \partial_{x}\right) \Psi, \\
\partial_{t_{2}} \Psi & =\left(\sum_{n=1}^{M-2} \lambda^{n} \partial_{\tau_{n}}+P_{M-3} \partial_{x}\right) \Psi,
\end{aligned}
$$

where we have $M+2$ variables $t_{1}, y_{1}, t_{2}, \tau_{k}, 1 \leqslant k \leqslant M-2, x$, and $P_{M-3}$ is a polynomial in $\lambda$ of the order $M-3$. Compatibility of this equations gives $(M+2)$-dimensional closed system for $v$ and coefficients of the polynomial $P_{M-3}$. 


\section{Quasiclassical SDYM type hierarchies as a re- duction of general multidimensional case}

In this section we will demonstrate that quasiclassical SDYM type hierarchies represent a simple and natural reduction of general linearly degenerate multidimensional dispersionless hierarchy. We define this reduction in terms of wave functions of polynomial distribution describing the hierarchy. We will introduce generating relations and the dressing scheme for the reduced hierarchy. The functional freedom in the reduced dressing scheme (number of variables of the functions defining the dressing data) doesn't change in the process of reduction, indicating that multidimensional universal hierarchy is of the same dimensionality as initial general case.

\subsection{Polynomial linearly degenerate multidimensional disper- sionless hierarchy}

Equations of the class we consider here are usually represented as commutation relations for a pair of vector fields (Lax pair) with coefficients holomorphic with respect to the spectral variable, they are usually called

dispersionless (or quasilinear) integrable equations. In general, vector fields contain a derivative over spectral variable (e.g., dispersionless KP equation). A picture of general multidimensional dispersionless integral hierarchy, starting from the properties of the wave functions, generating relation in terms of differential $N$-form and Lax-Sato equations, was developed in [12], 13]

In this work we consider Lax pairs with a spectral variable entering vector fields only as a parameter (there is no derivative over spectral variable in vector fields). We will use the term linearly degenerate for this case. Sometimes it is called weakly nonlinear, in twistor theory the term hyperCR case is also used. Typical representatives of this class are Plebanśki heavenly equations and equation (7).

First, we will outline the picture of linearly degenerate dispersionless hierarchy, using the description given in [13] for the general dispersionless hierarchy, starting from wave functions and generating differential $M$-form.

The involutive distribution of vector fields of codimension $M$ can be defined through the differential form [16], [17]

$$
\Omega_{M}=d \Psi^{1} \wedge d \Psi^{2} \wedge \ldots d \Psi^{M}
$$

where the functions $\Psi^{i}$ are level functions for integral surfaces of the distribution, i.e., integral surfaces are given by intersection of level surfaces for 
these functions. These functions are annulated by vector fields considered as differential operators, and thus they are solutions of linear equations of the hierarchy ('wave functions'). For the general hierarchy [13] the functions $\Psi^{i}$ depend on the set of times $\mathbf{t}$ and a spectral parameter $\lambda$, differentials do not contain $d \lambda$, and level functions (wave functions) are formal series in $\lambda$

$$
\begin{aligned}
& \Psi^{k}=\Psi_{0}^{k}+\widetilde{\Psi}^{k} \\
& \Psi_{0}^{k}=\sum_{n=0}^{\infty} t_{n}^{k} \lambda^{n}, \quad \widetilde{\Psi}^{k}=\sum_{n=1}^{\infty} \Psi_{n}^{k}\left(\mathbf{t}^{1}, \ldots, \mathbf{t}^{M}\right) \lambda^{-n},
\end{aligned}
$$

where $1 \leqslant k \leqslant M$, depending on $M$ infinite sequences of independent variables $\mathbf{t}^{k}=\left(t_{0}^{k}, \ldots, t_{n}^{k}, \ldots\right), t_{0}^{k}=x_{k}$.

The hierarchy is generated by the relation

$$
\left(J^{-1} d \Psi^{1} \wedge d \Psi^{2} \wedge \ldots d \Psi^{M}\right)_{-}=0
$$

where $(\cdots)_{-}$denotes the projection to the part of $(\cdots)$ with negative powers in $\lambda$ (respectively $(\cdots)_{+}$projects to nonnegative powers) and $J=\operatorname{det}\left(\partial_{j} \Psi^{i}\right)_{i, j=1, \ldots, M}$. Reduction $J=1$ corresponds to volume-preserving (divergence-free vector fields) case. Relation (22) implies Lax-Sato equations defining the dynamics of wave functions over higher times, moreover, it is equivalent to the set of Lax-Sato equations [13].

Generating relation represents a polynomiality condition for the coefficients of the form

$$
\widetilde{\Omega}_{M}=J^{-1} d \Psi^{1} \wedge d \Psi^{2} \wedge \ldots d \Psi^{M},
$$

leading to the polynomiality of integrable distribution corresponding to this form. This condition can be provided using Riemann-Hilbert problem (see below).

To construct polynomial vector fields belonging to the integrable distribution corresponding to relation (22), it is convenient to use the following observation [12]:

Proposition 1. For arbitrary vector field $\hat{V}$ in the space of variables $\mathbf{t}$ with coefficients polynomial in $\lambda$ and $\boldsymbol{\Psi}=\left(\Psi^{1}, \ldots, \Psi^{M}\right)$, where functions $\Psi^{i}$ of the form (21) satisfy generating relation (22),

$$
(\hat{V} \boldsymbol{\Psi})_{+}=\mathbf{0} \Longrightarrow \hat{V} \boldsymbol{\Psi}=\mathbf{0} .
$$

This observation gives an opportunity to construct polynomial vector fields belonging to the integrable distribution (linear operators of the hierarchy) in the spirit of Manakov ring technique [18], controlling only 'singular' 
part of the result of action of the operator on wave function (here it is a multicomponent function). First, we can introduce a basic set of vector fields $\hat{V}_{n}^{i}=\lambda^{n} \partial_{i}$, where $\partial_{i}=\frac{\partial}{\partial x_{i}}$, Then for arbitrary derivative over higher time $\partial_{n}^{k}=\frac{\partial}{\partial t_{n}^{k}}, n \geqslant 1$, there exists a unique linear combination of basic vector fields such that

$$
\left(\partial_{n}^{k} \Psi\right)_{+}=\left(\sum_{i, m} u_{m}^{i}(\mathbf{t}) \lambda^{m} \partial_{i} \Psi\right)_{+},
$$

and, due to Prop 1, we get linear equations of the hierarchy in the form

$$
\partial_{n}^{k} \Psi=\sum_{i, m} u_{m}^{i}(\mathbf{t}) \lambda^{m} \partial_{i} \Psi
$$

Introducing the Jacobian matrix

$$
\left(\mathrm{Jac}_{0}\right)=\left(\frac{D\left(\Psi^{1}, \ldots, \Psi^{M}\right)}{D\left(x_{1}, \ldots, x_{M}\right)}\right), \quad \operatorname{det}\left(\mathrm{Jac}_{0}\right)=J
$$

it is possible to write the hierarchy in the Lax-Sato form

$$
\left.\left.\partial_{n}^{k} \boldsymbol{\Psi}=\sum_{i=1}^{M}\left(\left(\mathrm{Jac}_{0}\right)^{-1}\right)_{i k} \lambda^{n}\right)\right)_{+} \partial_{i} \boldsymbol{\Psi}, \quad 1 \leqslant k \leqslant M,
$$

where $1 \leqslant n<\infty, \Psi=\left(\Psi^{1}, \ldots, \Psi^{M}\right)$.

First flows of the hierarchy (lowest level integrable distribution) read

$$
\partial_{1}^{k} \boldsymbol{\Psi}=\left(\lambda \partial_{k}-\sum_{p=1}^{M}\left(\partial_{k} u_{p}\right) \partial_{p}\right) \boldsymbol{\Psi}, \quad 1 \leqslant k \leqslant M,
$$

where $u_{k}=\Psi_{1}^{k}, 1 \leqslant k \leqslant M$. A commutativity for any pair of vector fields (24) (e.g., with $\partial_{1}^{k}$ and $\partial_{1}^{q}, k \neq q$ ) implies closed nonlinear (M+2)dimensional system of PDEs for the set of functions $u_{k}$, which can be written in the form

$$
\partial_{1}^{k} \partial_{q} \hat{U}-\partial_{1}^{q} \partial_{k} \hat{U}+\left[\partial_{k} \hat{U}, \partial_{q} \hat{U}\right]=0
$$

where $\hat{U}$ is a vector field, $\hat{U}=\sum_{p=1}^{M} u_{p} \partial_{p}$.

Higher flows of the hierarchy can be written also in remarkably simple recursive form

$$
\partial_{n}^{k} \boldsymbol{\Psi}=\left(\lambda \partial_{n-1}^{k}-\sum_{p=1}^{M}\left(\partial_{n-1}^{k} u_{p}\right) \partial_{p}\right) \boldsymbol{\Psi}, \quad 1 \leqslant k \leqslant M,
$$


which can be easily checked using Prop 1. Formally commuting a pair of vector fields (25) with distinct higher times, we get $(\mathrm{M}+4)$-dimensional quasiclassical SDYM equation

$$
\partial_{n}^{k} \partial_{l-1}^{q} \hat{U}-\partial_{l}^{q} \partial_{n-1}^{k} \hat{U}+\left[\partial_{n-1}^{k} \hat{U}, \partial_{l-1}^{q} \hat{U}\right]=0
$$

(compare to (3)), reflecting the fact that our generic $(\mathrm{M}+2)$-dimensional hierarchy can be naturally immersed into a special $(\mathrm{M}+4)$-dimensional hierarchy (see below).

\section{The dressing scheme}

It is easy to see that $\tilde{\Omega}_{M}$ is invariant under diffeomorphism

$$
\left(\Psi^{1}, \ldots, \Psi^{M}\right) \rightarrow \mathbf{F}\left(\lambda, \Psi^{1}, \ldots, \Psi^{M}\right)
$$

Let us consider functions $\Psi^{k}$ holomorphic inside and outside the unit circle (we denote components $\Psi_{\text {in }}^{k}$ and $\Psi_{\text {out }}^{k}, \Psi_{\text {out }}^{k}$ correspond to the series introduced above), having a discontinuity on it. If they satisfy a nonlinear vector Riemann-Hilbert problem (nvRHp)

$$
\left(\Psi^{1}, \ldots, \Psi^{M}\right)_{\text {in }}=\mathbf{F}\left(\lambda, \Psi^{1}, \ldots, \Psi^{M}\right)_{\text {out }},
$$

then the form $\tilde{\Omega}_{M}$ is holomorhic in all the complex plane.

Thus nvRHp gives a tool to construct forms $\tilde{\Omega}_{M}$, generating integrable distributions with holomorphic (meromorhic) coefficients and solutions to corresponding involution equations. See [11, [12], [13] for more detail, nonlinear vector $\bar{\partial}$ problem can be also used in the dressing scheme.

\subsection{Description of the class of reductions}

In this work we consider a class of reductions of general hierarchy (22), (23) characterised by the condition that $M-P, 1 \leqslant P<M$, of the series $\Psi^{k}$ are equal to 'vacuum' functions $\Psi_{0}^{k}=\sum_{n=0}^{\infty} t_{n}^{k} \lambda^{n}$ (for finite subsets of times they are polynomial)

$$
\left(\Psi^{k}\right)_{-}=0, \Psi^{k}=\Psi_{0}^{k}=\sum_{n=0}^{\infty} t_{n}^{k} \lambda^{n}, \quad P<k \leqslant M .
$$

Generating relation (22) for the reduced hierarchy takes the form

$$
\left(J^{-1} d \Psi^{1} \wedge \ldots d \Psi^{P} \wedge \Psi_{0}^{P+1} \wedge \ldots d \Psi_{0}^{M}\right)_{-}=0
$$


where the Jacobian is effectively taken for the set of functions $\Psi^{1}, \ldots, \Psi^{P}$.

Lax-Sato equations (23) have rather special structure in this case, taking into account that the only nonzero entries of last $M-P$ lines of the Jacobian form the unity matrix.

Reduction (27) is characterized rather simply in terms of the dressing scheme (26). The dressing data for this scheme are represented by the diffeomorphism F. Let us take a diffeomorphism that gives an identical transformation for last $M-P$ components,

$$
F^{k}\left(\lambda, \Psi^{1}, \ldots, \Psi^{M}\right)=\Psi^{k}, \quad P<k \leqslant M .
$$

Then the functions $\Psi^{k}, P<k \leqslant M$, don't have a discontinuity on the unit circle and are analytic in the complex plane, thus leading us to reduction (27). The Riemann-Hilbert problem is then written for the first $P$ functions $\Psi^{k}$,

$$
\Psi_{\text {in }}^{q}=F^{q}\left(\lambda, \Psi^{1}, \ldots, \Psi^{P}, \Psi_{0}^{P+1}, \ldots, \Psi_{0}^{M}\right)_{\text {out }}, \quad 1 \leqslant q \leqslant P
$$

The functional freedom of the dressing data is $p$ functions of $M+1$ variables, that indicates that reduced equations are generically $M+2$-dimensional, like equations of unreduced hierarchy.

However, if we consider the hierarchy with complete initially introduced set of times $\mathbf{t}^{\mathbf{1}}, \ldots, \mathbf{t}^{\mathrm{M}}$, we discover that the hierarchy contains subhierarchies with different dimensionalities (different number of independent variables in corresponding equations). Geometrically this is due to the fact that some of the level functions $\Psi^{i}$ defining integral surfaces for vector fields are rather special and depend only on some subset of the variables of the hierarchy (level functions $\Psi^{i}=\Psi_{0}^{i}$ ). Thus, we can choose some subsets of the variables of the hierarchy (finite or infinite), for which the effective number of level functions is less than $M$, then the codimension of corresponding distribution is less than $M$, and dimensionality of corresponding equations of the hierarchy (involutivity equations) is less than $M+2$. It would be interesting to study geometry of distributions for this hierarchy, which is probably described locally in terms of some special submanifolds of the Grassmannian [16]. However, in our examples we will try to choose special subsets of times to get rid of this degeneracy and obtain $M+2$-dimensional equations.

The case $M=4, P=2$ after additional divergence-free reduction leads to the six-dimensional heavenly equation [3]. For $P>2, M=P+2$ we get equation considered in [11, 4]. We are planning to discuss these cases in more detail later. 
The multidimensional reduced hierarchies considered in this work correspond to the choice $P=1$, i.e., we have one nontrivial function $\Psi$ and several vacuum functions $\Psi_{0}^{i}$. Vector fields in this case are effectively onedimensional, and corresponding hierarchies can be considered as multidimensional extensions of Shabat-Martínez Alonso universal hierarchy [9].

\section{$2.3 \quad$ Universal hierarchy}

Universal hierarchy of Shabat and Martínez Alonso [9] for 'positive' times

is a general hierarchy described in subsection 2.1 for $M=1$. In this case we have one wave function $\Psi$,

$$
\Psi=\Psi_{0}+\widetilde{\Psi}, \quad \Psi_{0}=\sum_{n=0}^{\infty} t_{n} \lambda^{n}, \quad \widetilde{\Psi}=\sum_{n=1}^{\infty} \Psi_{n}(\mathbf{t}) \lambda^{-n},
$$

generating equation (22) acquires the form

$$
\left(\Psi_{x}^{-1} d \Psi\right)_{-}=0
$$

and Lax-Sato equations are

$$
\partial_{n} \Psi=\left(\left(\Psi_{x}\right)^{-1} \lambda^{n}\right)_{+} \partial_{x} \Psi
$$

where $x=t_{0}, \partial_{n}=\frac{\partial}{\partial t_{n}}$. The first two flows

$$
\begin{aligned}
\partial_{y} \Psi & =\left(\lambda-v_{x}\right) \partial_{x}, \\
\partial_{t} \Psi & =\left(\lambda^{2}-v_{x} \lambda-v_{y}\right) \partial_{x},
\end{aligned}
$$

where $y=t_{1}, t=t_{2}, v=\Psi_{1}(\mathbf{t})$, give a Lax pair for $(2+1)$-dimensional equation (7),

$$
v_{x t}=v_{y y}+v_{x} v_{x y}-v_{x x} v_{y},
$$

discussed in the Introduction.

The Riemann-Hilbert problem (26) for the dressing scheme associated with universal hierarchy looks like

$$
\Psi_{\text {in }}=F\left(\lambda, \Psi_{\text {out }}\right) .
$$

The properties of solutions and inverse problem for equation (77) were recently studied in [19].

Lax-Sato equations (29) can be represented in recursive form (13),

$$
\left(\partial_{n}-\lambda \partial_{n-1}\right) \Psi=-\left(\partial_{n-1} v\right) \partial_{x} \Psi, \quad n \geqslant 1 .
$$




\subsection{Four-dimensional universal hierarchy}

Let us consider a reduction of the four-dimensional hierarchy (Section 2.1, $M=2$ ) characterised by the condition

$$
\Psi^{2}=\Psi_{0}^{2}
$$

We drop higher times $t_{n}^{1}, n>1$ to get rid of $(2+1)$-dimensional part of the hierarchy corresponding to universal hierarchy for these times and consider two wave functions

$$
\Psi=x+\sum_{n=1}^{\infty} \Psi_{n}(\mathbf{t}) \lambda^{-n}, \quad \Psi^{1}=\sum_{n=0}^{\infty} t_{n+1} \lambda^{n},
$$

generating relation (22) for this case reads

$$
\left(\Psi_{x}^{-1} d \Psi \wedge \sum_{n=0}^{\infty} \lambda^{n} d t_{n+1}\right)_{-}=0
$$

Lax-Sato equations of reduced hierarchy can be extracted from general LaxSato equations (23) and have a rather special structure

$$
\partial_{n+1} \Psi=\left(\lambda^{n} \partial_{y}-\left(\left(\Psi_{x}\right)^{-1} \Psi_{y} \lambda^{n}\right)_{+} \partial_{x}\right) \Psi
$$

where $y=t_{1}$. They can also be represented in a very simple recursive form, implied by Prop. 1,

$$
\left(\partial_{n+1}-\lambda \partial_{n}\right) \Psi=-\left(\partial_{n} v\right) \partial_{x} \Psi, \quad n \geqslant 1 .
$$

First two flows in recursive form

$$
\begin{aligned}
& \left(\partial_{t}-\lambda \partial_{y}\right) \Psi=-\left(v_{y}\right) \partial_{x} \Psi \\
& \left(\partial_{w}-\lambda \partial_{t}\right) \Psi=-\left(v_{t}\right) \partial_{x} \Psi
\end{aligned}
$$

where $t=t_{2}, w=t_{3}$, give a Lax pair for a four-dimensional equation

$$
v_{w y}=v_{t t}+v_{y} v_{x t}-v_{y x} v_{t} .
$$

Riemann-Hilbert problem for the dressing scheme associated with this case is

$$
\Psi_{\text {in }}=F\left(\lambda, \Psi_{\text {out }}, \sum_{n=0}^{\infty} \lambda^{n} t_{n+1}\right),
$$

and the dressing data is represented by the function of three variables $F(\lambda, X, Y)$, that corresponds to the functional freedom for Cauchy problem for equation (34). 


\section{Immersion of universal hierarchy into four-dimensional uni- versal hierarchy}

There exists a natural immersion of universal hierarchy into four-dimensional universal hierarchy. On the level of distributions it manifests itself in the fact that the basis (30) can be represented as the basis (33) plus one extra vector (for $n=0$ ). Codimension of distribution (30) is 1 , and codimension of distribution (33) equals 2, respective dimensionality of involutivity equations (equations of the hierarchy) is 3 and 4 . Having solutions of equations of universal hierarchy depending on some set of higher times, we obtain solutions of four-dimensional universal hierarchy.

This immersion has also a simple interpretation in terms of generating equation and dressing scheme. Let us have a solution of generating equation for universal hierarchy (28),

$$
\begin{aligned}
& \left(\Psi_{x}^{-1} d \Psi\right)_{-}=0, \\
& \Psi=\sum_{n=0}^{\infty} \lambda^{n} t_{n}+\widetilde{\Psi}, \quad \widetilde{\Psi}=\sum_{n=1}^{\infty} \Psi_{n}(\mathbf{t}) \lambda^{-n},
\end{aligned}
$$

then the function $\Psi^{\prime}=x+\widetilde{\Psi}$ satisfies generating equation (311) for fourdimensional universal hierarchy

$$
\left(\Psi_{x}^{\prime-1} d \Psi^{\prime} \wedge \sum_{n=0}^{\infty} \lambda^{n} d t_{n+1}\right)_{-}=0
$$

because, evidently,

$$
\left(\Psi_{x}^{\prime-1} d \Psi^{\prime} \wedge \sum_{n=0}^{\infty} \lambda^{n} d t_{n+1}\right)_{-}=\left(\Psi_{x}^{-1} d \Psi \wedge \sum_{n=0}^{\infty} \lambda^{n} d t_{n+1}\right)_{-}=0
$$

In terms of the dressing scheme the immersion corresponds to some special choice of the dressing data for the problem (35), namely

$$
F(\lambda, X, Y)=G(\lambda, X+\lambda Y),
$$

which allows to get (special) solutions of four-dimensional universal hierarchy from solutions of universal hierarchy.

\subsection{Five-dimensional universal hierarchy}

Let us take $P=1, M=3$ and consider the series

$$
\Psi=x+\sum_{n=1}^{\infty} \Psi_{n}(\mathbf{t}) \lambda^{-n}, \Psi^{1}=\sum_{n=0}^{\infty} t_{n+1}^{1} \lambda^{n}, \Psi^{2}=\sum_{n=0}^{\infty} t_{n+1}^{2} \lambda^{n},
$$


generating relation (22) for this case reads

$$
\left(\Psi_{x}^{-1} d \Psi \wedge \sum_{n=0}^{\infty} \lambda^{n} d t_{n+1}^{1} \wedge \sum_{n=0}^{\infty} \lambda^{n} d t_{n+1}^{2}\right)_{-}=0,
$$

and Lax-Sato equations (23) split into two copies of Lax-Sato equations for the four-dimensional universal hierarchy (32),

$$
\begin{aligned}
& \partial_{n+1}^{1} \Psi=\left(\lambda^{n} \partial_{y_{1}}-\left(\left(\Psi_{x}\right)^{-1} \Psi_{y_{1}} \lambda^{n}\right)_{+} \partial_{x}\right) \Psi, \\
& \partial_{n+1}^{2} \Psi=\left(\lambda^{n} \partial_{y_{2}}-\left(\left(\Psi_{x}\right)^{-1} \Psi_{y_{2}} \lambda^{n}\right)_{+} \partial_{x}\right) \Psi,
\end{aligned}
$$

where $y_{1}=t_{1}^{1}, y_{2}=t_{1}^{2}, \partial_{n}^{k}=\frac{\partial}{\partial t_{n}^{k}}$. Taking first two flows of this hierarchy, we get linear equations (44) and five-dimensional quasiclassical SDYM equation (5). Thus, equations (36) represent Lax-Sato equation of the hierarchy associated with five-dimensional quasiclassical SDYM equation.

Solutions to the hierarchy can be constructed using nonlinear RiemannHilbert problem

$$
\Psi_{\text {in }}=F\left(\lambda, \Psi_{\text {out }}, \sum_{n=0}^{\infty} t_{n+1}^{1} \lambda^{n}, \sum_{n=0}^{\infty} t_{n+1}^{2} \lambda^{n}\right)
$$

with the dressing data represented by the function of four variables.

The hierarchy can be also represented in recursive form

$$
\begin{gathered}
\partial_{n+1}^{1} \Psi=\left(\lambda \partial_{n}^{1}-\left(\partial_{n}^{1} v\right) \partial_{x}\right) \Psi \\
\partial_{n+1}^{2} \Psi=\left(\lambda \partial_{n}^{2}-\left(\partial_{n}^{2} v\right) \partial_{x}\right) \Psi,
\end{gathered}
$$

where $1 \leqslant n<\infty$.

\subsection{Multidimensional reduced hierarchy}

Let us consider multidimensional case $P=1, M>3$, corresponding to $M$ wave functions (with only $P=1$ nontrivial)

$$
\Psi=x+\sum_{n=1}^{\infty} \Psi_{n}(\mathbf{t}, x) \lambda^{-n}
$$

and

$$
\Psi^{k}=\sum_{n=0}^{\infty} t_{n+1}^{k} \lambda^{n}, \quad 1 \leqslant k \leqslant M-1
$$


Generating relation (22) for this case reads

$$
\left(\Psi_{x}^{-1} d \Psi \wedge \sum_{n=0}^{\infty} \lambda^{n} d t_{n+1}^{1} \wedge \cdots \wedge \sum_{n=0}^{\infty} \lambda^{n} d t_{n+1}^{M-1}\right)_{-}=0
$$

and Lax-Sato equations are constructed of the same building blocks as in the previous case,

$$
\partial_{n+1}^{k} \Psi=\left(\lambda^{n} \partial_{y_{k}}-\left(\left(\Psi_{x}\right)^{-1} \Psi_{y_{k}} \lambda^{n}\right)_{+} \partial_{x}\right) \Psi, \quad 1 \leqslant k \leqslant M-1, n \geqslant 1 .
$$

where $\partial_{n}^{k}=\frac{\partial}{\partial t_{n}^{k}}, y_{k}=t_{1}^{k}$. Recursive form of the hierarchy is

$$
\partial_{n+1}^{k} \Psi=\left(\lambda \partial_{n}^{k}-\left(\partial_{n}^{k} v\right) \partial_{x}\right) \Psi, \quad n \geqslant 1 .
$$

Solutions to the hierarchy can be constructed using nonlinear RiemannHilbert problem

$$
\Psi_{\text {in }}=F\left(\lambda, \Psi_{\text {out }}, \sum_{n=0}^{\infty} t_{n+1}^{1} \lambda^{n}, \ldots, \sum_{n=0}^{\infty} t_{n+1}^{M-1} \lambda^{n}\right)
$$

with the dressing data represented by the function of $(M+1)$ variables, that indicates that maximal dimensionality of equations of the hierarchy is $(M+2)$.

Each of Lax-Sato equations (37) is three-dimensional. To extract $(M+$ 2)-dimensional equation in the framework of this hierarchy, it is necessary to perform a restriction to some submanifold of variables to get rid of degeneracy. The geometric origin of this degeneracy was explained in subsection 1.1 , where we have demonstrated how to perform a restriction to submanifold and obtain 6-dimensional equation for the case $M=4$ (15). The scheme of restriction can be easily generalized to multidimensional case. Let us consider a system of lowest order Lax-Sato equations (37)

$$
\partial_{t_{k}} \Psi=\left(\lambda \partial_{y_{k}}-\left(\partial_{y_{k}} v\right) \partial_{x}\right) \Psi, \quad 1 \leqslant k \leqslant M-1,
$$

with the set of variables $t_{k}=t_{2}^{k}, y_{k}=t_{1}^{k}, x$. Codimension of distribution (38) is equal to $M$, which corresponds to the codimension of the hierarchy. Let us restrict this set of variables to $(M+2)$-dimensional submanifold

$$
\begin{aligned}
& y_{2}=t_{3}=\tau_{1}, \\
& \ldots \\
& y_{k+1}=t_{k+2}=(-1)^{k+1} \tau_{k}, \\
& \ldots \\
& y_{M-2}=t_{M-1}=(-1)^{M-2} \tau_{M-3}, \\
& y_{M-1}=(-1)^{M-1} \tau_{M-2}
\end{aligned}
$$


with the variables $x, t_{1}, t_{2}, y_{1}, \tau_{1}, \ldots, \tau_{M-2}$. Restriction of the distribution corresponding to equations (38) to the tangent space for this submanifold gives a 2-dimensional distribution (Lax pair)

$$
\begin{aligned}
& \partial_{t_{1}} \Psi=\left(\lambda \partial_{y_{1}}-\left(\partial_{y_{1}} v\right) \partial_{x}\right) \Psi, \\
& \partial_{t_{2}} \Psi=\left(\sum_{n=1}^{M-2} \lambda^{n} \partial_{\tau_{n}}+P_{M-3} \partial_{x}\right) \Psi,
\end{aligned}
$$

where $P_{M-3}$ is a polynomial in $\lambda$ of the order $M-3$. Compatibility of these equations (commutativity of vector fields) gives $(M+2)$-dimensional closed system for $v$ and coefficients of the polynomial $P_{M-3}$.

\section{Acknowledgements}

The authors are grateful to A.B. Shabat, V.E. Adler, B. Krulikov, A.V. Mikhailov and M. Dunajski for useful discussions. This work was partially supported by the RAS Presidium program 'Nonlinear dynamics in mathematical and physical sciences' and by the RFBR grant 14-01-00389.

\section{References}

[1] Mark J. Ablowitz, Sarbarish Chakravarty and Leon A. Takhtajan, A self-dual Yang-Mills hierarchy and its reductions to integrable systems in $1+1$ and $2+1$ dimensions, Communications in Mathematical Physics 158(2), 289-314 (1993)

[2] Maciej Dunajski, Solitons, instantons, and twistors, OUP Oxford (2009)

[3] Jerzy F. Plebański and Maciej Przanowski, The Lagrangian of a selfdual gravitational field as a limit of the SDYM lagrangian, Phys. Lett. A 212 (1-2), 22-28 (1996)

[4] S.V. Manakov, P.M. Santini, Inverse scattering problem for vector fields and the Cauchy problem for the heavenly equation, Phys. Lett. A $359(6), 613-619$ (2006)

[5] M.V. Pavlov, Integrable hydrodynamic chains, J. Math. Phys. 44(9) (2003) 4134-4156 
[6] E.V. Ferapontov, K.R. Khusnutdinova, On integrability of (2+1)dimensional quasilinear systems, Comm. Math. Phys., 248 (2004) 187206

[7] E.V. Ferapontov, K.R. Khusnutdinova, The characterization of 2component $(2+1)$-dimensional integrable systems of hydrodynamic type, J. Phys. A: Math. Gen., 37(8) (2004) 2949 - 2963

[8] E.V. Ferapontov, K.R. Khusnutdinova, C. Klein, On linear degeneracy of integrable quasilinear systems in higher dimensions, Letters in Mathematical Physics 96(1) 5-35 (2011)

[9] L. Martínez Alonso and A. B. Shabat, Energy-dependent potentials revisited: a universal hierarchy of hydrodynamic type, Phys. Lett. A 300 (2002) 58-64.

[10] Maciej Dunajski and George Sparling, A dispersionless integrable system associated to $\operatorname{Diff}\left(S^{1}\right)$ gauge theory, Phys.Lett. A343 (2005) 129132

[11] L.V. Bogdanov and B.G. Konopelchenko, On the $\bar{\partial}$-dressing method applicable to heavenly equation, Phys. Lett. A 345(1-3), 137-143 (2005)

[12] L.V. Bogdanov, V.S. Dryuma and S.V. Manakov, Dunajski generalization of the second heavenly equation: dressing method and the hierarchy, Journal of Physics A: Mathematical and Theoretical 40 (48), 14383 (2007)

[13] L.V. Bogdanov, A class of multidimensional integrable hierarchies and their reductions, Theoretical and Mathematical Physics 160 (1), 887$893(2009)$

[14] E.V. Ferapontov, K.R. Khusnutdinova, Hydrodynamic reductions of multidimensional dispersionless PDEs: The test for integrability, J. Math. Phys. 45 No. 6 (2004) 2365-2377.

[15] A. Sergyeyev, Recursion Operators for Multidimensional Integrable Systems, arXiv:1501.01955 [math.AP] (2015)

[16] L V Bogdanov and B G Konopelchenko, Grassmannians Gr(N-1, N+1), closed differential $\mathrm{N}$-1-forms and $\mathrm{N}$-dimensional integrable systems, Journal of Physics A: Mathematical and Theoretical 46 (8), 085201 (2013) 
[17] L V Bogdanov and B G Konopelchenko, Projective differential geometry of multidimensional dispersionless integrable hierarchies, Journal of Physics: Conference Series 482 (1), 012005 (2014)

[18] V.E. Zakharov and S.V. Manakov, Construction of higher-dimensional nonlinear integrable systems and of their solutions, Functional Analysis and Its Applications 19, Issue 2, pp 89-101 (1985)

[19] P G Grinevich, P M Santini and D Wu, The Cauchy problem for the Pavlov equation, Nonlinearity 28(11) (2015) 